\title{
COMPLEX AND FRACTAL COMPONENTS IN INDUSTRIAL DESIGN
}

\author{
N. SALA \\ Accademia di Architettura, Università della Svizzera Italiana, Mendrisio, Switzerland.
}

\begin{abstract}
Industrial design is an applied art where the aesthetics and the usability of products may be improved During the 20th century, we have seen an interesting transformation in our society from hand-made consumer goods designed and made by skilled craftsmen to mass production using new materials and technologies. Design aspects specified by the industrial designer may include the object's overall shape, the location of the details with respect to one another, colours, textures and ergonomics. Often, through the application of industrial design, a product's appeal to the consumer is greatly improved. Industrial design consists of the ideation of a shape or configuration, or composition of a pattern or colour. An industrial design can be a twoor three-dimensional pattern used to produce an object. For many years designers were inspired by Euclidean geometry and Euclidean shapes (e.g. triangles, squares, Platonic solids and polyhedra), and it is not surprising that industrial design objects have Euclidean characteristics. The evolution of materials (e.g. steel, plastic, glass) and technologies (from hand-made products to Computerized Numeric Control) have permitted designers to overcome the limits imposed by Euclidean geometry. Thus, modern design studies apply complex shapes and fractal geometry to create new kinds of objects that have futuristic shapes. The aim of this paper is to present some examples of industrial design objects that are analysed using the complexity and the fractal geometry.

Keywords: complexity, fractal geometry, industrial design, nature, self-similarity.
\end{abstract}

\section{INTRODUCTION}

In industrial design it is customary to look for the presence of mathematical and geometrical components (e.g. the golden ratio, the symmetry, the spirals, the curves, the surfaces and the Euclidean geometry) [1-5]. We can also observe the objects created by industrial design from a different point of view; for example, we can find some complex or fractal components that are present in the objects. In the present analysis, the complexity is not conceived as an area of chaos, but as an attempt to overcome the Euclidean shapes. The complexity is connected to the fractal geometry. Fractal geometry describes the irregular shapes and it can occur in many different places in both mathematics and elsewhere in nature. Fractal objects are irregular in shape, and they are generally self-similar and independent of scale. There are several mathematical structures that are fractals; for instance, the Koch snowflake [6], the Julia set [7] and the Lorenz attractor [8]. Complexity and fractal geometry can inspire an aesthetic sense. In fact, in the 1930s the mathematician George Birkhoff (1884-1944) proposed a measure of beauty defined as:

$$
M=\frac{O}{C}
$$

where $M$ represents the 'aesthetic measure' (or beauty), $O$ the order and $C$ the complexity. This measure suggests that beauty is related to order and complexity.

Complex and fractal components appeared in industrial design after the development of certain materials, e.g. the introduction of float glass, and manufacturing techniques, e.g. the work with the laser. This exploration and development of materials, manufacturing techniques and design are often 
indistinguishable from one another. In this paper, the complex and fractal analysis of industrial design has been organized into three parts:

1. the complex components in industrial design (e.g. the complex shapes and textures in the works of Alessandro Mendini);

2. the self-similarity in industrial design objects;

3. the box-counting dimension applied in industrial design objects to determine their degree of complexity.

\section{THE COMPLEX COMPONENTS IN INDUSTRIAL DESIGN}

A new kind of industrial design was born owing to the complexity. To investigate the complex components in industrial design objects, we can study the complex covers (e.g. in the works of Alessandro Mendini) and the complex surfaces (e.g. in Cini Boeri's monolithic chair realized in float glass or Zaha Hadid's objects created for Alessi) derived from the observation of nature and natural objects.

The Italian architect and designer Alessandro Mendini conceived the armchair Proust (1978), for Studio Alchimia, with bright colour and quirky ornamentation. The complexity of the cover, shown in Fig. 1, recalls the drip paintings realized by Jackson Pollock (1912-1956).

Mendini conceived, for Alessi, a tea and coffee service (2003) made of wood carved by hand with 925/1000 silver inside. We can observe the complex shapes present in Mendini's project in the shape of the teapot in Fig. 2a, which is similar to a shell with fractal decoration shown in Fig. 2b [9]. Mendini's tea and coffee service has been realized for Alessi's 'Tea \& Coffee Towers' project,

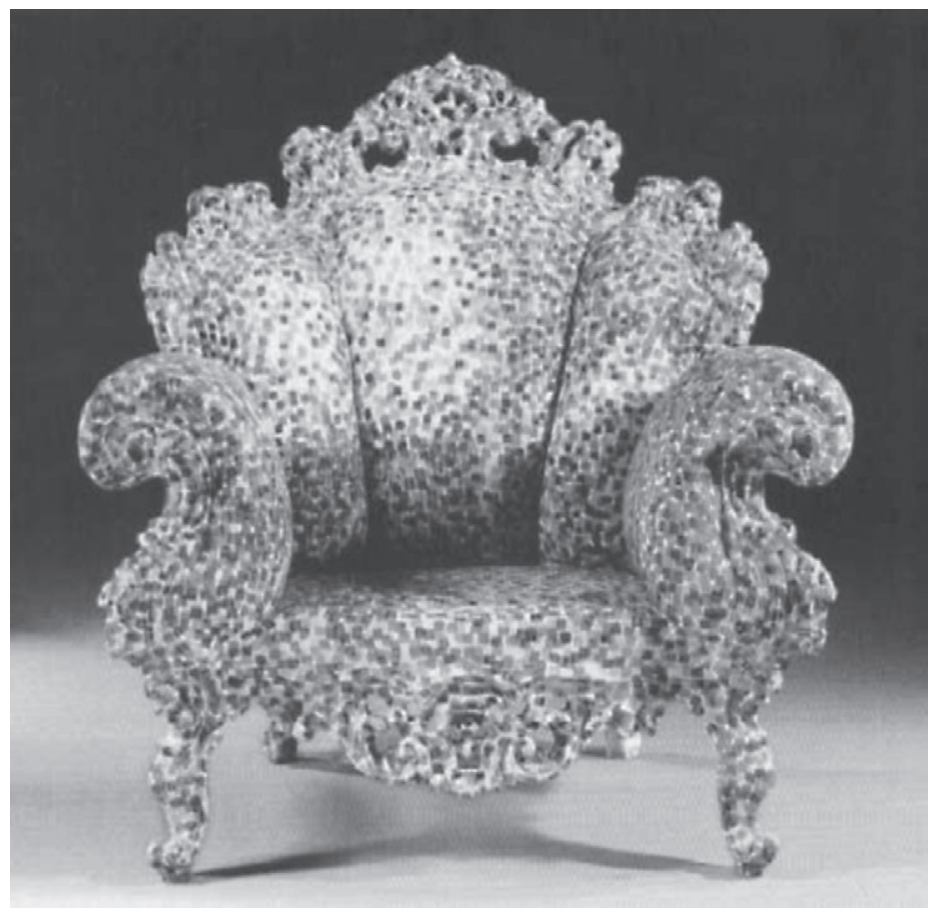

Figure 1: Proust (1978), conceived by Mendini for Studio Alchimia, showing the complexity of the cover as in Pollock's paintings. 
a new landscape of objects designed by contemporary architects [10]. This project combines the expression of architecture with the language of design. Zaha Hadid has created for Alessi's 'Tea \& Coffee Towers' project a coffee-tea pot in 925/1000 silver (Fig. 3) that recalls futuristic and fractal shapes. Hadid used 'broken' symmetry and fractal geometry to realize her projects [9]. Observing Figs 3 and 4, we can note the complexity of the shapes as a three-dimensional puzzle, the single parts form the unity. This object exploits the combination and the contrast between the vertical and the horizontal parts [10].

The architect and designer Cini Boeri created, in collaboration with Tomu Katayanagi, Ghost (1987) - a curved float glass monolithic chair, $12 \mathrm{~mm}$ thick - for FIAM Italia (Italy). The shape, shown in Fig. 5, is smooth and complex, and it has been realized using a special manufacturing process created by FIAM Italia.

Complex components in industrial design are also generated by observing nature, plants and animals. Ross Lovegrove designed a new PET bottle (1999-2002) for Ty Nant based on the fluidity
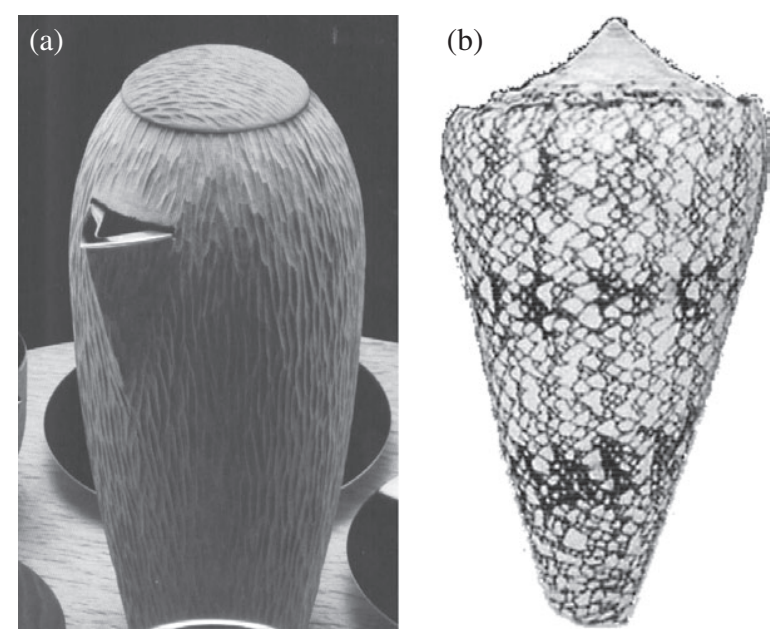

Figure 2: Tea and coffee service (2003) conceived by Mendini for Alessi (a) showing complex decorations like a shell (Conus araneosus) (b).

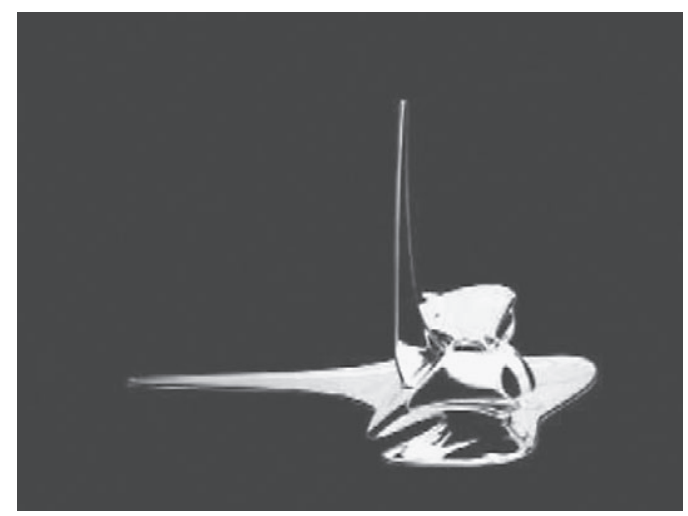

Figure 3: Tea and coffee service (2003) created by Hadid for Alessi. 


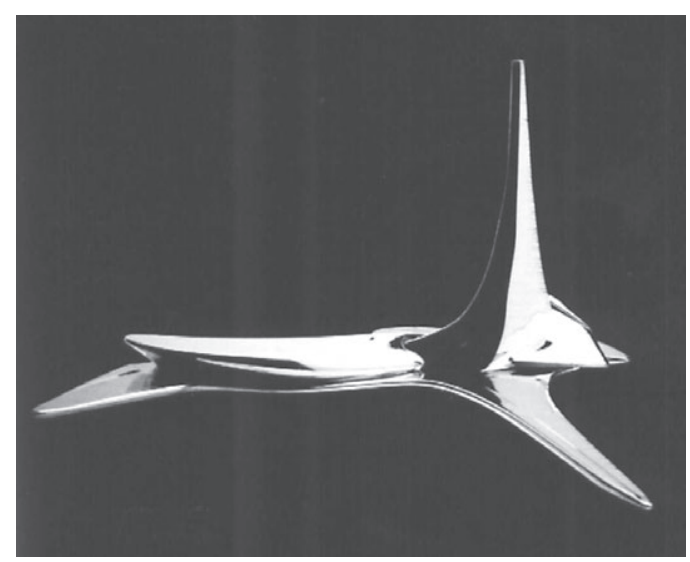

Figure 4: Tea and coffee service (2003), created by Hadid for Alessi, exploiting the combination and the contrast between the vertical and the horizontal parts.

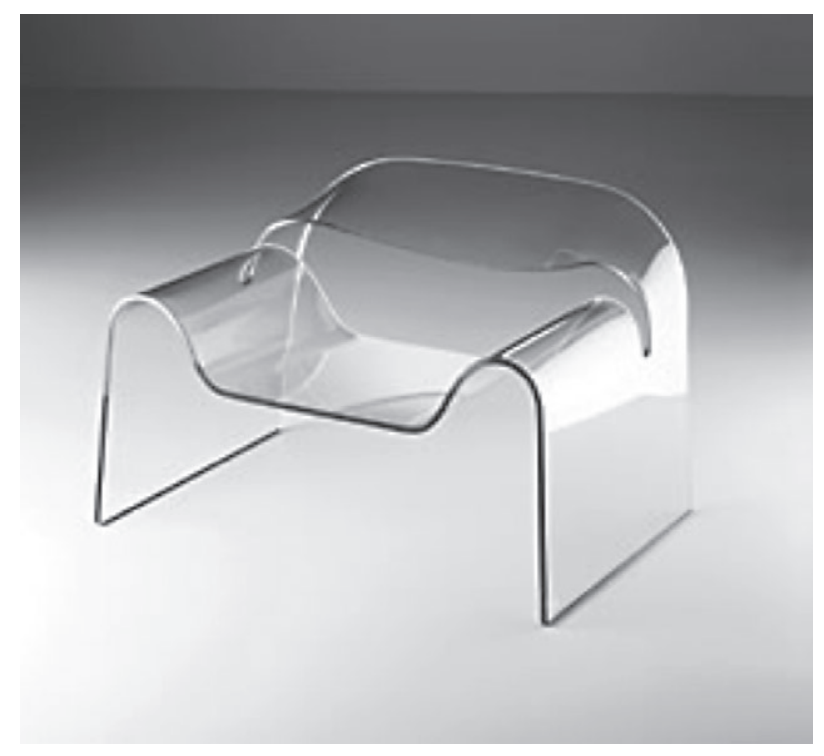

Figure 5: Ghost (1987), conceived by Cini Boeri for FIAM Italia, showing a complex shape.

of water. The bottle is conceptually unique in terms of production delivery and in form to the water concept, demonstrating advances in blow moulding techniques. As the bottle has a fluid asymmetrical form, the surface has an irregular and contoured shape which evokes the fluidity of water.

Danish architect and designer Poul Henningsen (1894-1967) is widely regarded as a true 'master of lamp making'. Two of the most prevalent icons of Henningsen's career are the PH5 lamp, designed in 1924, and the PH Artichoke (1958). The PH5 lamp, also known as the Paris lamp for its award winning appearance at the Paris World Exhibition, used tiers of shades, enabling the user to direct light in several different directions without exposing the bulb. The PH Artichoke, shown in Fig. 6a, is 

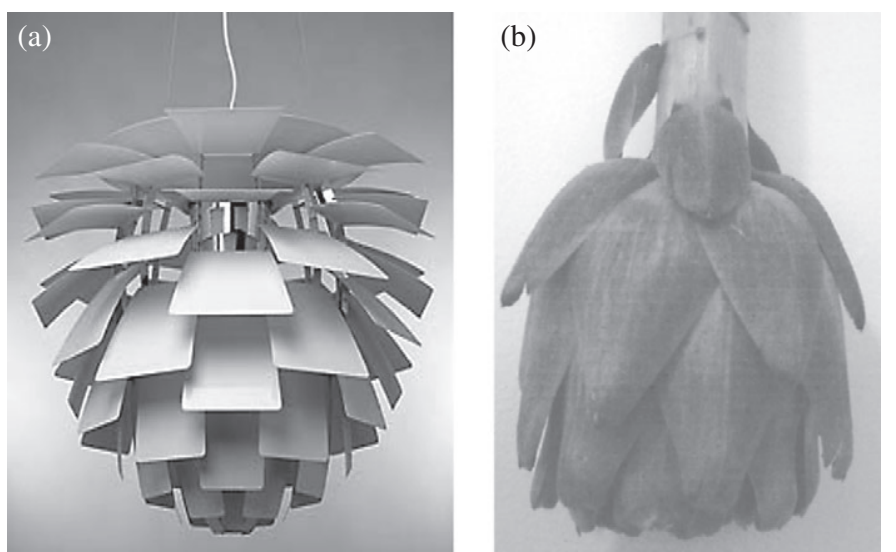

Figure 6: PH Artichoke (1958) conceived by Henningsen for Poulsen (a) similar to an artichoke (b).
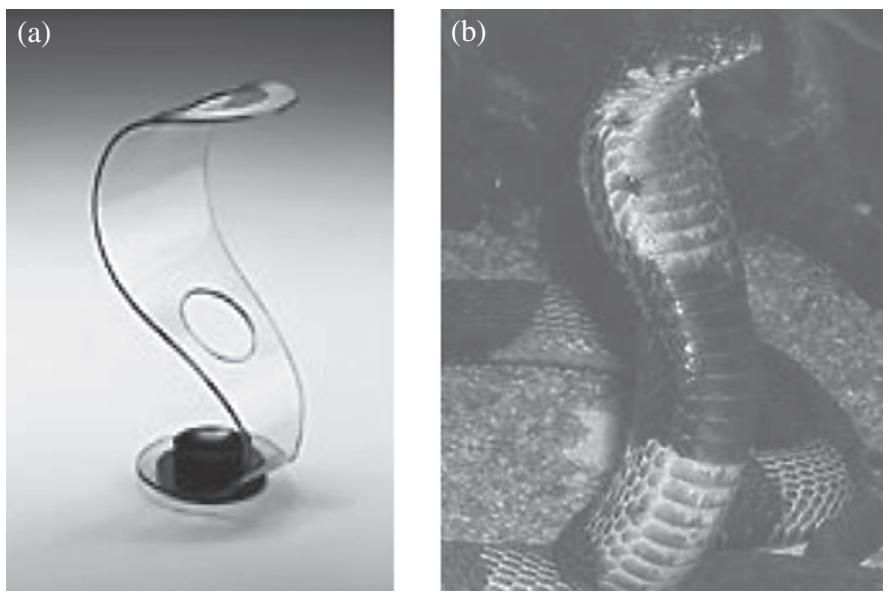

Figure 7: COBRA (1991) umbrella stick stand conceived by Vigna for FIAM (a) similar to a real cobra (b).

based on the same principle, although it had even more panels and layers of shades. This Henningsen object is similar to a real artichoke (Fig. 6b) - the vegetable that inspired the lamp.

COBRA (1991) realized by the Italian designer Elio Vigna, Fig. 7a, is a curved float glass umbrella stick stand, $10 \mathrm{~mm}$ thick, inserted in a metallic black stained base. The similarity to a cobra snake (Fig. 7b) is remarkable.

Ravello (2002) is an armchair that was initially conceived by Oscar Niemeyer, a poetic gesture, and implemented by Ricardo Antonio for Poltrona Frau (Italy). Its structure is made of metal, the seat and the backrest are padded with polyurethane foam, and it is covered with Colour System Frau Leather. This flower-shaped armchair, shown in Fig. 8a, recalls a snowdrop (Fig. 8b). The citrus basket Blow up (2004) and the centrepiece bowl Blow Up (2004), realized by Humberto Campana and Fernando Campana for Alessi, shown in Fig. 9a and b, are other examples of industrial design objects that involve complex shapes which mimic the objects realized using natural materials common in African and Brazilian cultures. These very complex shapes are realized in 18/10 mirror-polished stainless steel. 


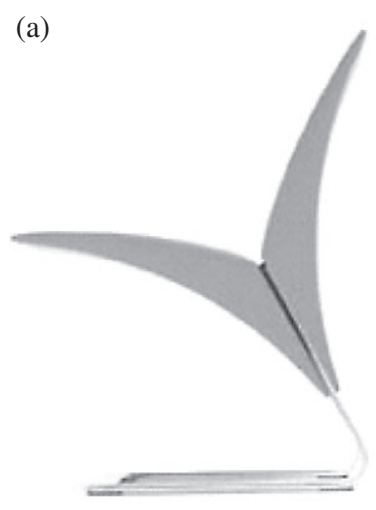

(b)

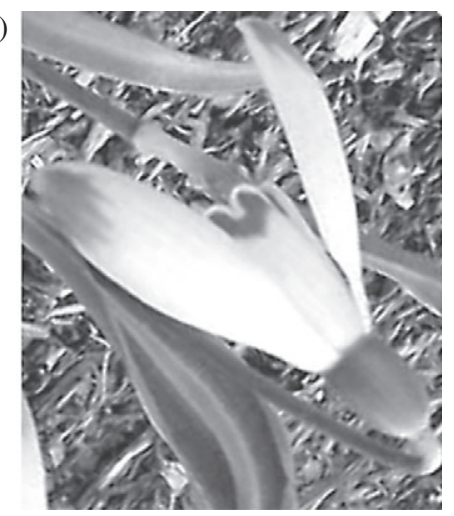

Figure 8: Ravello (2002) flower-shaped armchair conceived by Oscar Niemeyer and implemented by Ricardo Antonio for Poltrona Frau (a); a snowdrop (b).
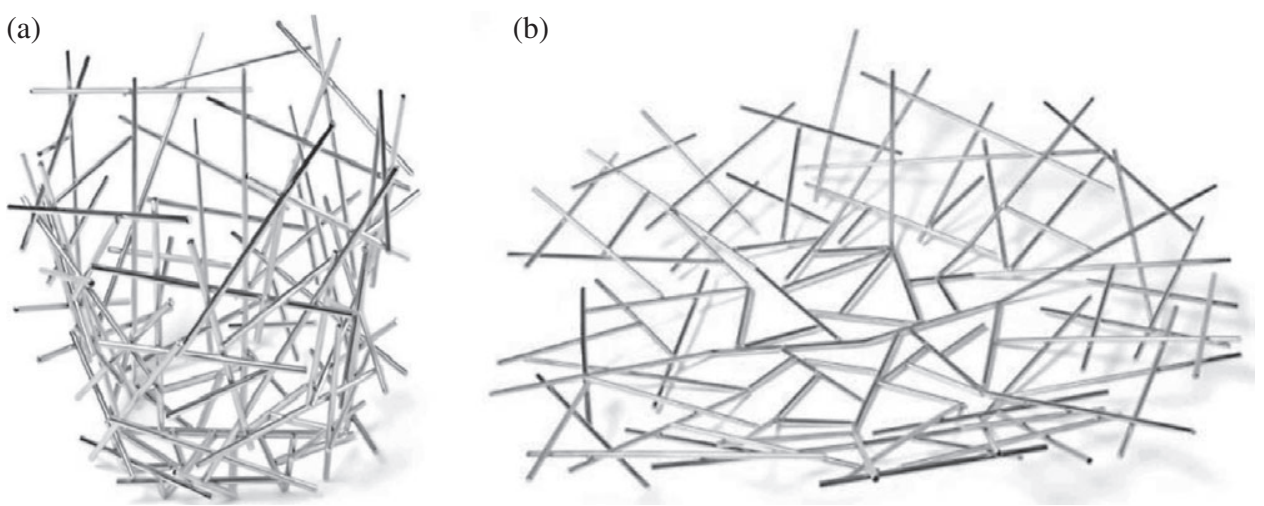

Figure 9: Citrus basket Blow up (2004) (a) and centrepiece bowl Blow Up (2004) (b) designed by Humberto and Fernando Campana for Alessi.

3 THE SELF SIMILARITY IN INDUSTRIAL DESIGN OBJECTS

Fractal geometry is a modern mathematical theory that departs radically from traditional Euclidean geometry. It describes objects that are scale symmetric, or self-similar. This means that when such objects are magnified, their parts are seen as an exact resemblance to the whole, the property continues with the parts of the parts and so on to infinity. These shapes are called fractals, and they must maintain a rough, jagged quality at every scale at which an object can be examined. The nature and the characteristics of fractals are reflected in the word itself, coined by the Polish-born French mathematician Benoit B. Mandelbrot (b. 1924) from the Latin verb frangere, 'to break', and from the related adjective fractus, 'fragmented and irregular' [11-14]. The acceptance of the word 'fractal' was dated in 1975, when Mandelbrot presented the list of publications between 1951 and 1975, the date when the French version of his book was published. A fractal object can be self-similar if it has undergone a transformation whereby the dimensions of the structure are all modified by the same scaling factor. The new shape may be smaller, larger, translated and/or rotated, but its shape remains similar. 'Similar' means that the relative proportions of the shapes' sides and internal angles remain the same. Our senses, having evolved in nature's self-similar cascade, appreciate 


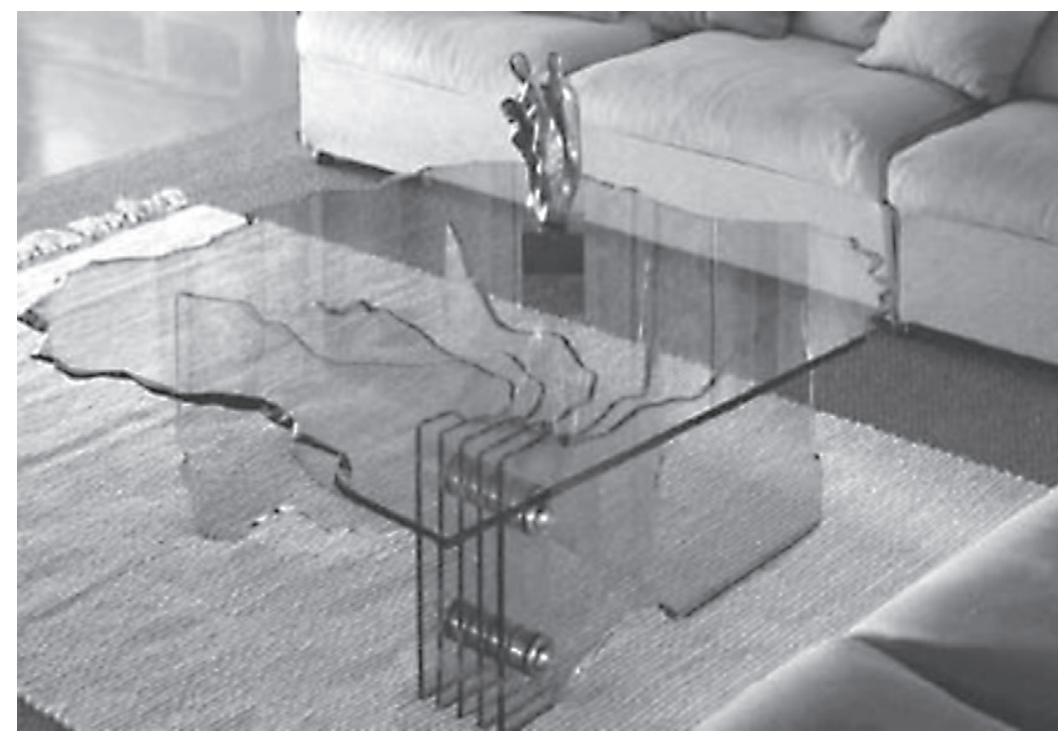

Figure 10: The coffee table Shell (1988) designed by Lane for FIAM mimics the irregular shapes present in nature.

self-similarity in designed objects [15]. Irregularity, self-similarity between the original structure and its smaller constitutive fragments, form invariance under changes of measure (scaling) and iteration of unit generators are the main properties which characterize fractal objects. Mandelbrot used the term 'self-similar' for the first time in 1964, in an internal report at IBM, where he was conducting research, and in the title of a 1965 paper. A fractal object is self-similar. This means that as we peer deeper into the fractal image, we can observe that the shapes seen at one scale are similar to the shapes seen in the detail at another scale. It is possible to demonstrate that fractal shapes and self-similarity were known to artists in different cultures [15].

In industrial design, we can observe self-similar shapes; for example, the American artist Danny Lame realized Shell (1988) for FIAM (Italy) (Fig. 10). It is a coffee table with hand-sculptured float glass top $15 \mathrm{~mm}$ thick. The base is made of five $12 \mathrm{~mm}$ thick hand-sculptured float glass elements, four of which curved. All the elements are kept together by stainless steel hardware. Lame's coffee table is a clear example of a design object that mimics the fractal-like components present in nature, in particular the 'broken' symmetry and the self-similarity.

The coffee table Papilio (1985) realized by Mendini for Zanotta (Italy) has fractal-like shapes. The table has two or three $8 \mathrm{~mm}$ shelves shaped and made from hand-cut tempered glass. The legs are made of brushed, nickel-plated steel, screwed onto stainless steel discs fixed to the glass. Figure 11 shows the images of the top that emphasize the irregularity. Figure 12 illustrates the two version of this coffee table.

The repetition in different scales is also present in Alessi's 'Tea \& Coffee Tower' project. The architect and designer Tom Kovac, Australian of Slovene descent, has realized for Alessi a tea and coffee service in double wall 925/1000 silver. Kovac is the Creative Design Director of Curvedigital, a joint project between RMIT University and Melbourne Museum. He has explored the potential of new processes and developments in non-standard manufacturing. The set of objects that make up this service is a kind of code which activates a geometric progression, shown in Fig. 13. 

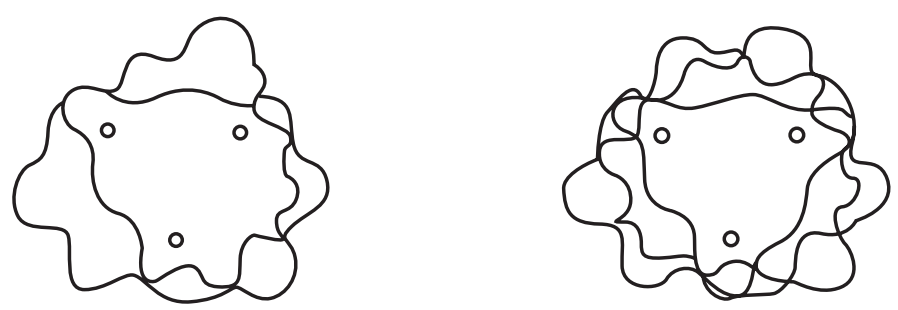

Figure 11: Coffee table Papilio (1985) realized by Mendini for Zanotta; the images of the top show irregular and fractal-like components.
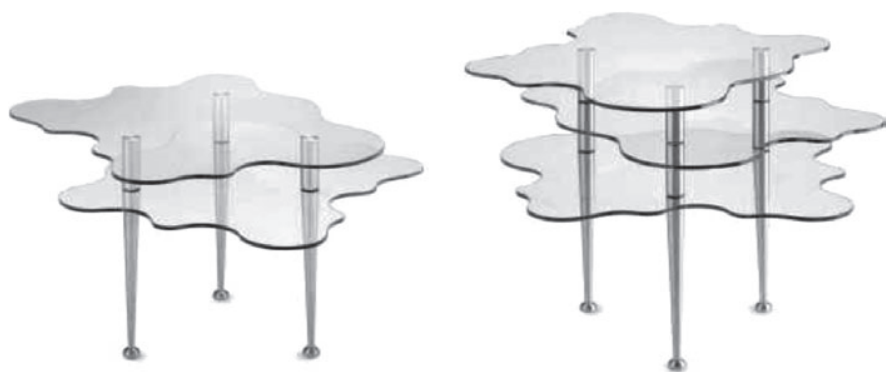

Figure 12: Papilio (1985) by Mendini, emphasizing the self-similarity.
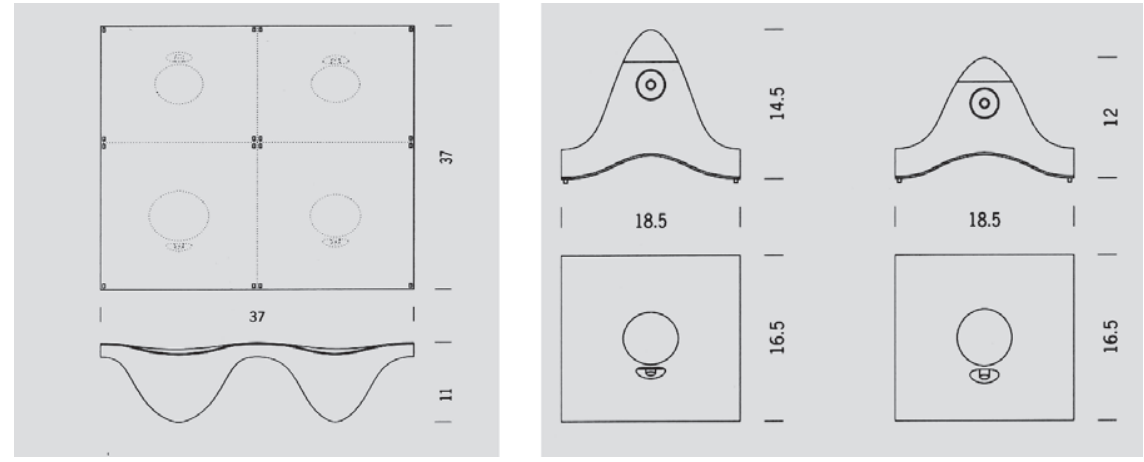

Figure 13: Tea and coffee service realized by Kovac for Alessi.

This progression opens a new hypothesis in the design's methodologies. The parts that form the set come from correlation criteria, by an inference from the conceptual level to the operating level, using various scales of representation [10]. Observing Figs 13 and 14, the properties of the self-similarity are evident and repeated in four different scales.

The self-similarity is also present in the cover of the chairs realized by the designer Paul Smith, shown in Fig. 15a. Figure 15b illustrates a detail of a Mandelbrot set of the level $\alpha(c)$ for $c \in M$, based on Alfred Barten's experiment [7]. The analogy between Fig. 15a and b reveals that creativity has found inspiration from mathematics. 


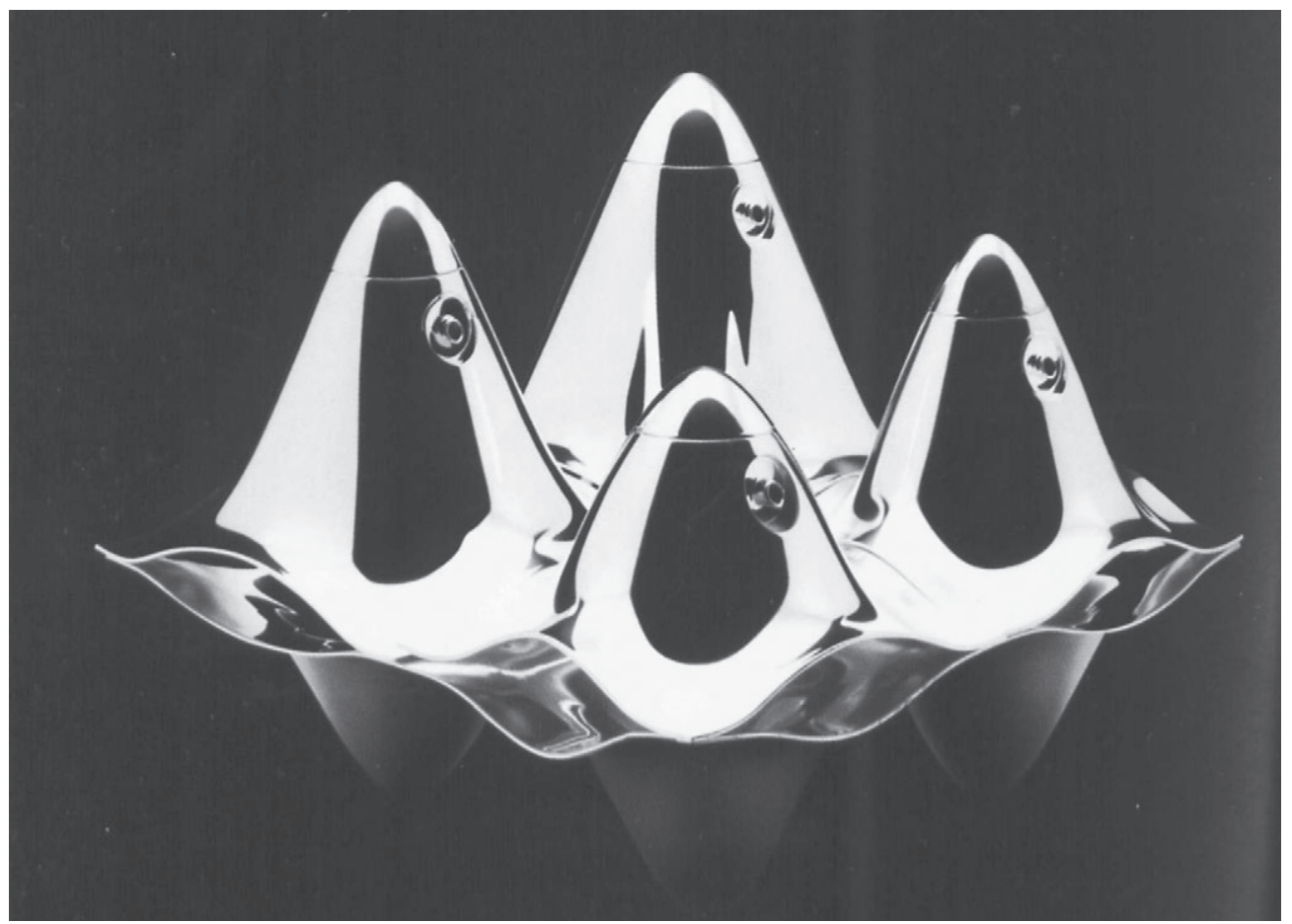

Figure 14: Tea and coffee service realized by Kovac for Alessi, showing the self-similarity applied in four different scales.

(a)

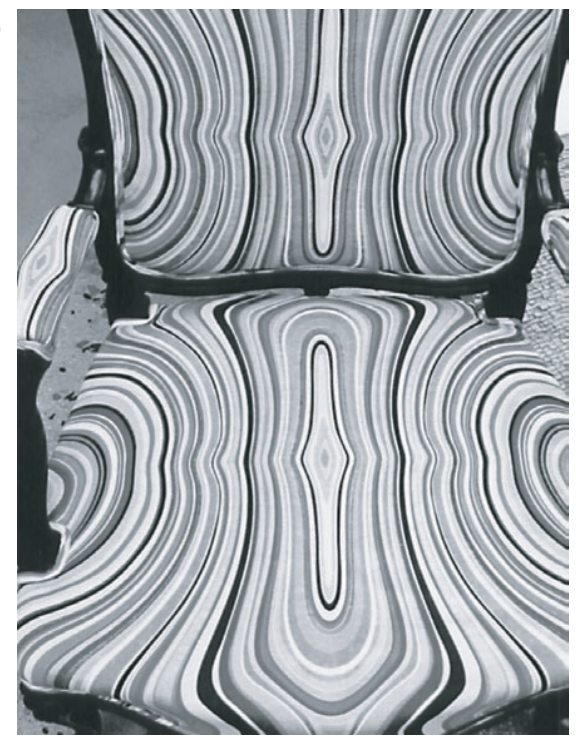

(b)

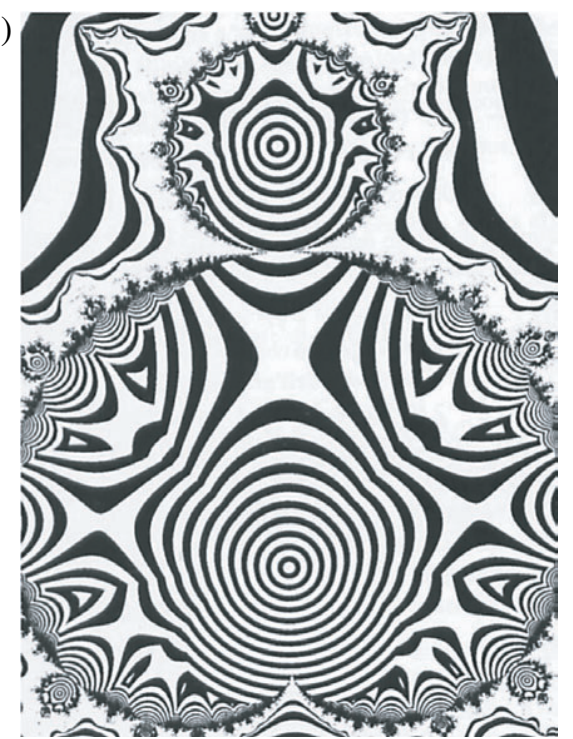

Figure 15: The self-similarity present in the cover of this chair (a) and in a fractal set (b). 


\section{THE BOX-COUNTING DIMENSION}

The box-counting dimension is connected to the problem of determining the fractal dimension of a complex two-dimensional image. It is defined as the exponent $D_{\mathrm{b}}$ in the relationship:

$$
N(d) \approx \frac{1}{d^{D_{\mathrm{b}}}}
$$

where $N(d)$ is the number of boxes of linear size $d$ necessary to cover a data set of points distributed in a two-dimensional plane. The basis of this method is that, for objects that are Euclidean, eqn (2) defines their dimension. One needs a number of boxes proportional to $1 / d$ to cover a set of points lying on a smooth line, proportional to $1 / d^{2}$ to cover a set of points evenly distributed on a plane and so on. Applying logarithms to eqn $(2)$, we obtain: $N(d) \approx-D_{\mathrm{b}} \log (d)$.

The box-counting dimension can be determined using the iterative procedure outlined below:

- $\quad$ superimpose a grid of square boxes over the image (the grid size is given as $s_{1}$ );

- count the number of boxes that contain some of the image $\left[N\left(s_{1}\right)\right]$;

- repeat this procedure, changing $s_{1}$ to a smaller grid size $s_{2}$;

- count the resulting number of boxes that contain the image $\left[N\left(s_{2}\right)\right]$;

- repeat this procedures changing $s$ to smaller and smaller grid sizes.

The box-counting dimension is defined by:

$$
D_{\mathrm{b}}=\frac{\left[\log \left(N\left(s_{2}\right)\right)-\log \left(N\left(s_{1}\right)\right)\right]}{\left[\log \left(N\left(1 / s_{2}\right)\right)-\log \left(N\left(1 / s_{1}\right)\right)\right]},
$$

where $1 / s$ is the number of boxes across the bottom of the grid. We can apply the box-counting dimension in industrial design too. It is calculated by counting the number of boxes that contain lines from the drawing inside them. Figure 16 illustrates the procedure to determine the complexity of a fern using the box count [9]. The image of a fern is created using computer algorithms based on the iterated function systems.

Using eqn (3) to determine the box-counting dimension of the fern shown in Fig. 16, we have:

$$
\begin{aligned}
D_{\mathrm{b}} & =\frac{\left[\log \left(N\left(s_{2}\right)\right)-\log \left(N\left(s_{1}\right)\right)\right]}{\left[\log \left(N\left(1 / s_{2}\right)\right)-\log \left(N\left(1 / s_{1}\right)\right)\right]}=\frac{[\log (90)-\log (27)]}{[\log (8)-\log (4)]}=1.736 \ldots, \\
D_{\mathrm{b}} & =\frac{\left[\log \left(N\left(s_{2}\right)\right)-\log \left(N\left(s_{1}\right)\right)\right]}{\left[\log \left(N\left(1 / s_{2}\right)\right)-\log \left(N\left(1 / s_{1}\right)\right)\right]}=\frac{[\log (315)-\log (90)]}{[\log (16)-\log (8)]}=1.807 \ldots .
\end{aligned}
$$

The box-counting dimension of the fern, calculated using eqn (3), is an irrational value between 1.736 and 1.807 . To determine the degree of complexity of industrial design objects, we can apply the iterative procedure presented above, and we can also use software solutions, the procedure for which is as follows:

- collect the images of the industrial design objects;

- digitize the images using a printer-copier;

- $\quad$ save them in bitmap (*.bmp) format;

- analyse the images using a software tool (e.g. the program Benoit ${ }^{\circledR}$, version 1.3 ) in order to calculate box $\left(D_{\mathrm{b}}\right)$, information $\left(D_{\mathrm{i}}\right)$ and mass dimensions $\left(D_{\mathrm{M}}\right)$, as well as their respective standard errors and intercepts on $\log -\log$ plots.

Using this procedure we calculated the box-counting dimension of two industrial design objects that evidence a complex shape: the mat Blow up (2004), realized by Humberto and Fernando Campana 

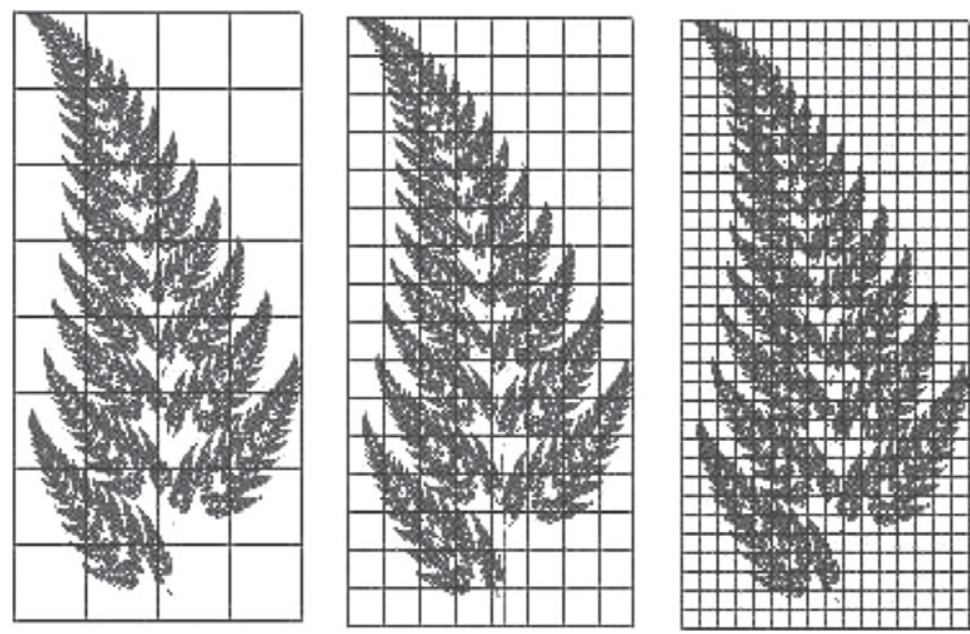

Figure 16: Box-counting dimension applied to a fern.
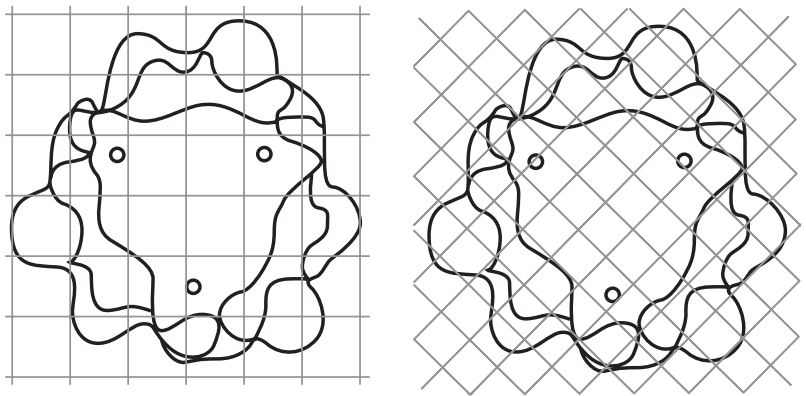

Figure 17: Box-counting method applied to the coffee table Papilio.

Table 1: Fractal analyses of industrial design objects.

\begin{tabular}{lccc}
\hline $\begin{array}{l}\text { Industrial } \\
\text { design object }\end{array}$ & $\begin{array}{c}\text { Box-counting } \\
\text { dimension }\end{array}$ & $\begin{array}{c}\text { Information } \\
\text { dimension }\end{array}$ & \multicolumn{1}{c}{$\begin{array}{c}\text { Mass } \\
\text { dimension }\end{array}$} \\
\hline $\begin{array}{l}\text { Mat Blow up } \\
(2004)\end{array}$ & $1.95727 \pm 0.0100442$ & $1.93884 \pm 0.00071467$ & $1.94470 \pm 0.0144529$ \\
$\begin{array}{l}\text { Papilio (1985), } \\
\text { two shelves }\end{array}$ & $1.88114 \pm 0.0079822$ & $1.86924 \pm 0.0003498$ & $1.88721 \pm 0.0011540$ \\
$\begin{array}{l}\text { Papilio }(1985), \\
\text { three shelves }\end{array}$ & $1.87092 \pm 0.0044575$ & $1.83010 \pm 0.0006803$ & $1.87370 \pm 0.0012665$ \\
\hline
\end{tabular}

for Alessi, and the coffee table Papilio (1985), designed by Mendini. Figure 17 shows the boxcounting method applied for 10 iterations to the coffee table Papilio. Table 1 presents the results of our analyses. 


\section{CONCLUSIONS}

The complexity paradigm is developing two different traditions: one in industrial design and the other in science. In this work we describe an approach where the complex and the fractal components have been analysed using three different points of view: to look for the complex components in an industrial design object, to determine the self-similarity in industrial design objects and to find the box-counting dimension applied in the design objects, to determine their degree of complexity. The examples described for points 2 and 3 introduce the concept of non-linear design. Non-linear design has influenced the work of designers such as Zaha Hadid, Marc Newson, Tom Kovac and other designers. In Alessi's 'Tea \& Coffee Tower' project there is an isomorphism between the complexity in architecture (complexity in the large scale) and the complexity in industrial design (complexity in the small scale). All the designers involved in this project, namely, Zaha Hadid, Massimiliano Fuksas and Doriana O. Mandrelli, Vito Acconci, Tom Kovac, Toyo Ito, Ben van Berkel and Caroline Bos, and others, worked on a dizzy change of scale and on some very different functions [10]. Except in the design, complexity and contradiction have been acknowledged everywhere, from Gödel's proof of ultimate inconsistency in mathematics to T.S. Eliot's analysis of 'difficult' poetry and Joseph Albers' definition of the paradoxical quality of 'painting' [16, p. 16]. In this century, we have observed the joint evolution of materials, processes and designs, and how through this mutual exploration, great products with complex shapes have emerged [17-25]. The complex components connected to the fractal geometry have reached maturity and they will influence the industrial design of the future.

\section{REFERENCES}

[1] Alessi, A. (ed.), Alessi: The Design Factory, Wiley Academy: New York, 1998.

[2] Fiell, C. \& Fiell, P., Design du XX $X^{e}$ siècle, Taschen: Köln, 2000.

[3] Munari, B., La scoperta del triangolo, Zanichelli: Bologna, 1976.

[4] Munari, B., La scoperta del quadrato, Zanichelli: Bologna, 1987.

[5] Elam, K., Geometry of Design, Princeton Architectural Press: New York, 2001.

[6] von Koch, H., Sur une courbe continue sans tangente, obtenue par une construction géometrique élémentaire. Arkiv för Matematik, 1, pp. 681-704, 1904.

[7] Peitgen, H.-O. \& Richter, P.H., The Beauty of Fractals: Images of Complex Dynamical Systems, Springer-Verlag: Berlin, 1986.

[8] Crilly, J., Earnshaw, R.A. \& Jones, H., Fractals and Chaos, Springer-Verlag: New York, pp. 43-69, 1991.

[9] Sala, N. \& Cappellato, G., Architetture della complessità, Franco Angeli: Milano, 2004.

[10] Mendini, A., Tea \& Coffee Towers, Electa: Milano, 2003.

[11] Mandelbrot, B.B., Les Objects Fractals. Forme, Hasard et Dimension, Flammarion: Paris, 1975.

[12] Peitgen, H.O., Hurgens, H. \& Saupe, D., Chaos and Fractals: New Frontiers of Science, Springer-Verlag: New York, 1992.

[13] Fivaz, R., L'ordre et la volupté, Press Polytechniques Romandes: Lausanne, 1988.

[14] Briggs, J., Fractals, the Patterns of Chaos, Thames and Hudson: London, 1992.

[15] Sala, N., Fractal geometry in the arts: an overview across the different cultures. Thinking in Patterns: Fractals and Related Phenomena in Nature, ed. M.M. Novak, World-Scientific: Singapore, pp. 177-188, 2004.

[16] Venturi, R., Complexity and Contradiction in Architecture, The Museum of Modern Art: New York, 1992.

[17] Bangert, A. \& Armer, K.M., 80s Style: Designs of the Decade, Abbeville Press: New York, 1990.

[18] Bayley, S. (ed.), The Conran Directory of Design, Villard Books: New York, 1985. 
[19] de Noblet, J. (ed.), Industrial Design: Reflections of a Century, Flammarion/APCI: Paris, 1993.

[20] Ashby, M. \& Johnson, K., Material and Design, Butterworth Heinemann: UK, 2002.

[21] Bejan, A., Advanced Engineering Thermodynamics, 2nd edn, Wiley: New York, 1997.

[22] Bejan, A., Shape and Structure, from Engineering to Nature, Cambridge University Press: Cambridge, UK, 2000.

[23] Hernandez, G., Allen, J.K. \& Mistree, F., Design of hierarchic platforms for customizable products, ASME Paper DECT2002/DAC-34095. Proc. of DETC'02, ASME 2002 Design Engineering Technical Conferences and Computer and Information in Engineering Conference, Montreal, Canada, 29 September-2 October 2002.

[24] Carone, M.J., Williams, C.B., Allen, J.K. \& Mistree, F., An application of constructural theory in multi-objective design of product platforms, ASME Paper DECT2003/DTM-48667. Proc. of DETC'03, ASME 2003 Design Engineering Technical Conferences and Computer and Information in Engineering Conference, Chicago, Illinois, 2-6 September 2003.

[25] Rosa, R.N., Reis, A.H. \& Miguel, A.F., Bejan's Constructal Theory of Shape and Structure, Évora Geophysics Center, University of Évora, Portugal, 2004. 